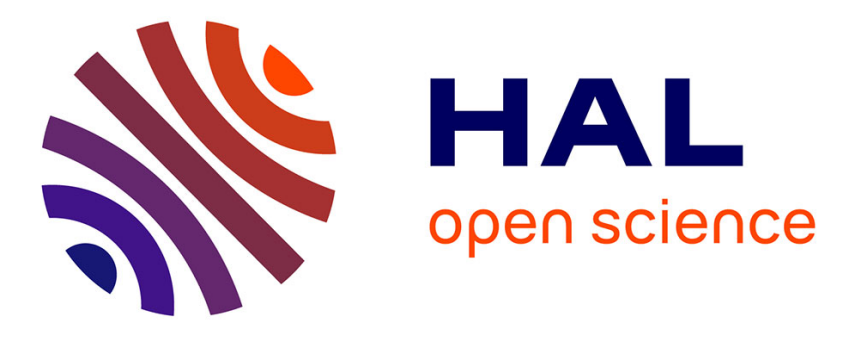

\title{
New Orbit Determination and Clock synchronisation modules for EGNOS
}

Anne Rouanet Labé, Rabab Lembachar, Thierry Authié, Sébastien Trilles, Flavien Mercier

\section{- To cite this version:}

Anne Rouanet Labé, Rabab Lembachar, Thierry Authié, Sébastien Trilles, Flavien Mercier. New Orbit Determination and Clock synchronisation modules for EGNOS. ION GNSS+ 2016, Sep 2016, Portland, United States. pp. 1-8. hal-01371836

\section{HAL Id: hal-01371836 https://hal.science/hal-01371836}

Submitted on 26 Sep 2016

HAL is a multi-disciplinary open access archive for the deposit and dissemination of scientific research documents, whether they are published or not. The documents may come from teaching and research institutions in France or abroad, or from public or private research centers.
L'archive ouverte pluridisciplinaire HAL, est destinée au dépôt et à la diffusion de documents scientifiques de niveau recherche, publiés ou non, émanant des établissements d'enseignement et de recherche français ou étrangers, des laboratoires publics ou privés. 


\section{Open Archive TOULOUSE Archive Ouverte (OATAO)}

OATAO is an open access repository that collects the work of Toulouse researchers and makes it freely available over the web where possible.

This is an author-deposited version published in: http://oatao.univ-toulouse.fr/ Eprints ID: 16117

To cite this version: Rouanet Labé, Anne and Lembachar, Rabab and Authié, Thierry and Trilles, Sébastien and Mercier, Flavien New Orbit Determination and Clock synchronisation modules for EGNOS. (2016) In: ION GNSS+ 2016, 12 September 2016 - 13 September 2016 (Portland, United States).

Any correspondence concerning this service should be sent to the repository administrator: staff-oatao@ listes-diff.inp-toulouse.fr 


\title{
New Orbit Determination and Clock synchronisation modules for EGNOS
}

\author{
A. Rouanet Labé, R. Lembachar, T. Authié, S. Trilles, Thales Alenia Space, France \\ F. Mercier, CNES, France
}

\section{BIOGRAPHIES}

Anne Rouanet Labé graduated from the Ecole Polytechnique France. She currently works as navigation algorithms engineer at Thales Alenia Space on the EGNOS project, more precisely on precise orbit determination.

Rabab Lembachar graduated from ENSEEIHT, France. She is a software engineer in the EGNOS Navigation Algorithms team at Thales Alenia Space, currently working on designing and conceiving an Orbit Determination software.

Thierry Authié graduated from the Institut National des Sciences Appliquées (INSA), France. He worked in space flight dynamics and precise orbit determination. He currently works as navigation algorithms engineer at Thales Alenia Space on the EGNOS project.

Sébastien Trilles is a specialist in navigation performances, working at Thales Alenia Space on the EGNOS Project as Algorithm Navigation Design Authority. He has been in charge of Algorithm studies with the CNES on Precise Orbit Determination and Time System Reference for EGNOS.

Flavien Mercier is senior expert in the orbit determination team at CNES. He works on the precise orbit determination for altimetry satellites (TOPEX, JASON) since 2001, he is specialist in GPS processing. $\mathrm{He}$ is also involved in validations for the EGNOS team. Since 2010, he is participating to the CNES/CLS analysis centre for IGS ('grg' products).

\section{ABSTRACT}

The purpose of a Satellite Based Augmentation System (SBAS), such as EGNOS or WAAS, is to identify all range error sources and to distribute the corresponding corrections to the civil aviation user community with reliable navigation services for different flight phases. The effect of a satellite location error depends on the user location while a satellite clock error with respect to a reference time scale directly translates into a common pseudorange error to all the users. Therefore the SBAS shall broadcast a $3 \mathrm{D}$ vector that represents the satellite orbit error and a satellite clock correction.

To achieve this objective the SBAS shall internally estimate the orbits and clocks for all the navigation satellites in view of the service area. The orbit determination function is in charge of computing the satellite ephemerides. The synchronization function computes the corresponding clock bias for each epoch and each satellite. Then the corrections are constructed from the differences between these orbits and clocks and the corresponding ones broadcasted inside the GNSS navigation messages.

Starting from R\&D activities, Thales Alenia Space has developed new orbit determination and synchronization modules that are part of the Thales Algorithm Navigation Chain. These modules have been designed in collaboration with the orbit determination team at CNES (the French Space Agency).

The new proposed orbit determination module is based on real time processing using code carrier measurement only. This module provides a stable and metric GPS orbit performance using an SBAS set of receivers corresponding to the EGNOS service area. The new synchronization module solves clock errors directly steered to GPS reference time scale, for the stations and satellites. It uses both code carrier and phase carrier measurements as well as the orbits estimated by the orbit determination process. The clocks solution error Allan's deviation is around $10^{-12}$ at $120 \mathrm{~s}$ leading to $7 \mathrm{~cm}$ of possible deviation for a prediction up to $120 \mathrm{~s}$. This performance is fully compatible with the needs of the SBAS mission.

These modules are now fully integrated into the SPEED platform, the SBAS Operational Test-bed that fully represents EGNOS Performances in terms of accuracy, continuity, availability and integrity for Safety Of Life services. The performance evaluation shows a real improvement over the current EGNOS algorithms, 
particularly in terms of the distribution of the Satellite Residual Error for the Worst user location (SREW).

This paper provides a high level architecture description of this new Thales solution. A set of performance figures showing the achieved improvements is also presented.

\section{INTRODUCTION}

Orbit and clock determination processes are key components of a SBAS, as they provide corrections to the GNSS signals models so as to improve the user positioning accuracy. A good precision in the determination of orbits and clocks induces lesser pseudorange errors for the user. This, in turn, allows the SBAS to broadcast lesser UDRE for the same integrity margins, which enhances the service levels availability.

As R\&D activity, in collaboration with the CNES, TAS has designed and implemented a new navigation chain to compute the GNSS signal corrections to be broadcasted by a SBAS, and in particular the GNSS orbit and clock determination processes. Their design is presented below. The performance of these modules is shown, the quality of the estimated orbits with respect to SP3 orbits, and the clock stability through the Allan variance.

The performance of these modules is assessed globally by looking at the pseudorange error a user in the service area will have using the orbit and clock corrections transmitted by the SBAS.

\section{REAL TIME ORBIT DETERMINATION AND PREDICTION FOR EGNOS}

The purpose of the orbit estimation module is to provide the GPS positions in the near future of the current epoch. These positions will be used to provide precise orbit corrections to EGNOS users.

A classical approach is to perform an orbit determination using a sufficiently long measurement dataset (a few orbits, with measurements processed using a Least Squares filter, this is the implementation used in the legacy EGNOS ground segment), and then extrapolate the orbit to provide the predicted GPS positions. However this approach has some drawbacks: the orbit determination arcs must be updated often depending on the possible extrapolation performance (typically every hour, for arcs adjusted on more than one day); the important shift between successive arcs will increase the discontinuities in the predicted GPS positions at each update; also the cpu charge is important for one arc orbit determination and leads to very important cpu charge fluctuations over time.

Using a sequential filter (recursive formulation for the Least Square filter) is an efficient way to obtain in real time the best possible extrapolated orbit, and also avoids the reprocessing of all measurements common between two successive orbit determination arcs. However, it is necessary to limit the time horizon of the adjusted orbit in this case, this is achieved by an exponential weighting applied on the past measurements contribution. This corresponds to the exponentially age-weighting approach in [2]. The filter is equivalent to an extended Kalman filter, except that there is no model noise, this is replaced by the exponential weighting strategy.

The next paragraphs show the measurements characteristics, the dynamic models used, the filter formulation and the corresponding state vector.

\section{A. Dynamic models}

The orbit determination process first needs to propagate the satellite orbits. For this, the main forces applied on a satellite have been implemented. They have been selected based on their amplitude on a GNSS orbit (Table 1).

\begin{tabular}{|l|l|}
\hline \multicolumn{1}{|c|}{ Force } & \multicolumn{1}{c|}{ Description } \\
\hline \hline $\begin{array}{l}\text { Earth gravity } \\
\text { potential }\end{array}$ & Modeled as spherical harmonics \\
\hline $\begin{array}{l}\text { Sun gravity } \\
\text { potential }\end{array}$ & Uses JPL Ephemerides from DE-405 \\
\hline $\begin{array}{l}\text { Moon gravity } \\
\text { potential }\end{array}$ & Uses JPL Ephemerides from DE-405 \\
\hline $\begin{array}{l}\text { Solid Earth } \\
\text { tides }\end{array}$ & Follow the IERS standard 2010 \\
\hline $\begin{array}{l}\text { Solid polar } \\
\text { tides }\end{array}$ & Follow the IERS standard 2010 \\
\hline $\begin{array}{l}\text { Direct solar } \\
\text { radiation } \\
\text { pressure }\end{array}$ & $\begin{array}{l}\text { Satellite modeled as a sphere. A shadow } \\
\text { function is used for eclipses modelling }\end{array}$ \\
\hline $\begin{array}{l}\text { Relativistic } \\
\text { force }\end{array}$ & $\begin{array}{l}\text { Three terms are computed: Schwarzchild, } \\
\text { Lense-Thirring and de Sitter }\end{array}$ \\
\hline Y-bias & Force acting along the solar panel axis \\
\hline
\end{tabular}

Table 1: List of forces acting on the GNSS satellite modeled in the orbit determination process

The dynamic equations are integrated in an inertial frame, namely the GCRF (Geocentric Celestial Reference Frame). The frames definition and conversions were implemented following the IERS standards 2010, and the use of IERS bulletins for the polar motion and UT1-UTC corrections.

\section{B. Measurement models}

Due to the required accuracy, dual frequency measurements are used (iono-free combination). Most precise solutions use pseudo-range and phase measurements, for example at IGS [3]. However, the use of the phase may be not necessary for intermediate precisions. The use of pseudo-range smoothed by the phase (for example using a Hatch filter) is also not necessary as the orbit determination process averages globally the measurements, so an intermediate average produced by the smoothed pseudo-range has no efficiency. 
Using the phase is not straightforward as it is necessary to adjust the ambiguities, and construct the passes without cycle slips. It is also necessary to manage the ambiguities on the fly, to suppress them from the state vector when a pass is terminated.

Thus we use only the iono-free combination of pseudoranges for the orbit determination. The measurement $m$ between station $i$ and satellite $j$ is modeled as follows [3]:

$$
m=\rho+h_{i}-h^{j}+T_{v} f(e l)+\varepsilon
$$

Where:

- $\rho$ is the geometric distance between the station center of phase at reception time, and the satellite center of phase at transmission time,

○ $h_{i}$ is the station clock offset,

○ $h^{j}$ is the satellite clock offset,

- $\quad T_{v}$ is the zenith tropospheric delay (ZTD)

- $f(e l)$ is the mapping function (the slant tropospheric delay is $T_{v} f(e l)$ ), the mapping function defined in the MOPS [1] is used.

$\circ \quad \varepsilon$ is the code measurement noise.

In precise measurement processing, there are separate models for hydrostatic and wet tropospheric delays. Here we use a simpler approach, as the required performance is not so stringent as for IGS products. The periodic relativistic clock correction term for the satellites [3] is included in $\rho$, to be compliant with the clocks definitions in the broadcasted orbits.

For the satellite center of phase offsets, no specific model is used. This simplifies the satellite modeling, as no attitude yaw steering is needed, so no geometrical model is used which avoid the monitoring and updating by the ground segment configurations.

\section{State vector}

For an orbit determination using iono-free pseudo-range measurements and long arcs (a few orbits), the adjusted parameters are :

- dynamical models: for each satellite, initial position and velocity, solar radiation pressure coefficient, $y$ bias acceleration.

- measurement models: satellite and station clocks at each epoch, zenith tropospheric delay for each station.

Using this set of parameters, the size of the problem to solve is small, except for the contribution of the clocks, one clock offset parameter at each epoch is to be adjusted. But these parameters are uncoupled between successive epochs (they are present in the measurement equations only for one epoch), so it is possible to eliminate them when processing sequentially the measurement equations in time. This is an advantage for the recursive filter, which can eliminate completely the clock offset parameters of the current epoch, when all corresponding measurements are processed.

The state vector for the recursive filter is described in Table 1:

\begin{tabular}{|c|l|l|c|}
\hline Parameter & \multicolumn{1}{|c|}{ Description } & \multicolumn{1}{|c|}{ Number } & Type \\
\hline \hline$X^{j}$ & $\begin{array}{l}\text { Position and } \\
\text { velocity of a } \\
\text { GNSS satellite }\end{array}$ & $\begin{array}{l}\text { x number } \\
\text { of GNSS } \\
\text { satellites }\end{array}$ & Dynamics \\
\hline$C_{s r p}$ & $\begin{array}{l}\text { Solar radiation } \\
\text { pressure } \\
\text { coefficient }\end{array}$ & $\begin{array}{l}1 \text { x number } \\
\text { of GNSS } \\
\text { satellites }\end{array}$ & Dynamics \\
\hline$Y_{\text {bias }}$ & $\begin{array}{l}\text { Amplitude of } \\
\text { the force acting } \\
\text { along the Y axis } \\
\text { of a GPS }\end{array}$ & $\begin{array}{l}1 \text { number } \\
\text { of GNSS } \\
\text { satellites }\end{array}$ & Dynamics \\
\hline$h^{j}$ & $\begin{array}{l}\text { Satellite clock } \\
1 \text { x number } \\
\text { of GNSS } \\
\text { satellites }\end{array}$ & Measurement \\
\hline$h_{i}$ & $\begin{array}{l}\text { Station clock } \\
\text { of number } \\
\text { stations }\end{array}$ & Measurement \\
\hline$T_{v}$ & $\begin{array}{l}\text { Vertical } \\
\text { tropospheric } \\
\text { delay }\end{array}$ & $\begin{array}{l}1 \text { number } \\
\text { of RIMS } \\
\text { stations }\end{array}$ & Measurement \\
\hline
\end{tabular}

Table 2: List of estimated parameters

In equation 1, we observe that there is a global unobservability at each epoch related to the clocks offsets.

This means that the clocks cannot be solved as is, since if a set of clocks is a solution, the same set plus an arbitrary value is also a solution. It is therefore necessary to add constraint equations on the clocks in order to fix this value. The chosen solution is to constrain loosely the satellite clocks to their values given by the GPS broadcast navigation messages. The weight of these constraint equations is much lower than the weight of the pseudorange measurement equations, the orbit solution is not impacted by this constraint.

\section{Filter formulation}

A recursive Square Root Information Filter formulation has been used (SRIF [4]). In the SRIF formulation, the information matrix $R_{k-1}$ (root square of the inverse covariance matrix), computed at epoch $k-1$ is added to the measurement equations to be processed at epoch $k$. This matrix $R_{k-1}$ contains all information over the past for the state vector $X_{k-1}$ (before epoch $k-1$ including this epoch). The clock contributions are eliminated, because there will be no measurements in the future depending directly on these clock terms (equation 1).

Then this information matrix is propagated with the corresponding state vector to epoch $k$. The clock terms in $X_{k}$ are reinitialized. The propagated information matrix is then multiplied by a coefficient slightly lower than 1.0 in order to limit the contribution of the past measurements to the current solution (exponential weighting method, 1.0 
would be the case of an arc containing all past measurements with identical weightings, so with a possible very long duration, not compatible with the dynamic model performance).

The SRIF formulation has the advantage to have a better condition number than a standard least squares algorithm (as for the Kalman Filter, the square root formulations like Bierman algorithm). Therefore, a greater numerical stability is achieved.

\section{E. Maneuver management}

The filter is designed to compute a solution based on a theoretical model (dynamic) and measurements, and to adjust a confidence associated to each source (covariance matrix). If one of the inputs (model or measurements) is slowly deteriorating, the residuals (differences between measurements and model) will increase.

A maneuver of a GPS satellite is a sudden change of the orbit which may cause rapid and huge divergence between model and measurements, especially if the satellite is not visible during the maneuver: the wrong orbit may be propagated by the dynamical process for hours, which involves huge orbit errors when new measurements are performed on this satellite (hundreds of kilometers). The filter cannot deal with this sudden jump on the orbit, and will surely diverge.

The system of maneuver management is composed of two steps: detection, based on the study of measurement residuals, and reset of the parameters of the satellite.

The detection of a maneuver is complementary to the elimination of outliers. If a measurement residual exceeds a threshold, there are two possibilities: wrong measurement (outlier) or wrong model (maneuver). In that last case, all the residues for the satellite will be impacted, and the measurements cannot be questioned.

When a maneuver is detected, the satellite is removed from the problem and the corresponding parameters are re-initialized. This avoids contamination to the others satellites.

The former orbit and the covariance matrix of the satellite are forgotten. A new orbit -position, velocity and covariance matrix- is roughly estimated through the measurements to ensure the filter to be as close as possible to a linear condition. This process is independent from the filter. As soon as the satellite's orbit is updated, the satellite is reintegrated in the global filter, with a high magnitude covariance matrix.

To summarize, three subjects must be particularly examined: discriminating maneuvers from outliers, fast detection of maneuver to avoid contamination to others satellites, and soft reinsertion of the satellite in the global filter after the independent reset.

\section{F. Performance results}

The following figure shows a comparison between 3 different orbit solutions with respect to the IGS orbit, on a 4 day time span. The errors are plotted in a Local Orbital Frame, respectively in radial, tangential, and normal components. The red curve is given by the EGNOS solution, it is not continuous as this solution was built using the slow correction messages, which are valid only when the satellite is in view of the service area.

The green curve is the solution calculated by the new orbit determination process.

The blue curve is given by the GPS navigation message. One can see that the new solution performs much better than EGNOS, and has a metric precision.
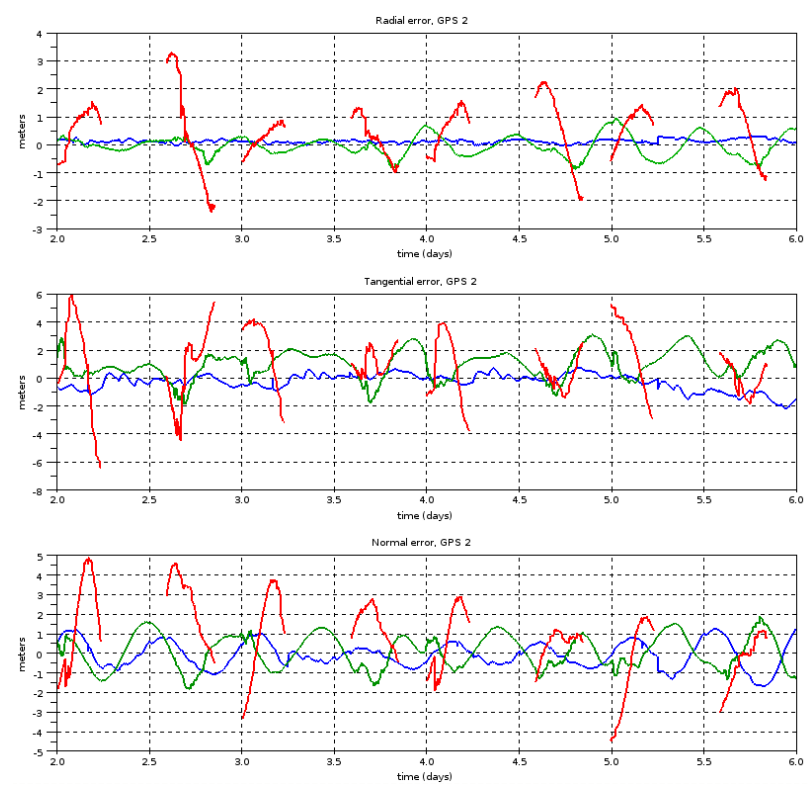

Figure 1: Error with respect to the SP3 for 3 orbit solutions: red: EGNOS, green: TAS solution, blue: GPS broadcast orbit

The maneuver detection system and the filter behavior are shown in the next figures. In this case, a GPS satellite has been maneuvering outside the visibility area of EGNOS RIMS stations, its orbit was then only propagated by the process until it comes back into visibility. When it did, the measurements showed that its position was far from where it was propagated. The filter reacted to this and a new orbit is estimated after reinitializing the satellite covariance.

On Figure 2, Figure 3, and Figure 4, the error between the estimated position and the IGS orbit is plotted in a Local Orbital Frame. The curve is blue when the satellite has no measurement and is only propagated, red when it has measurements but the estimated orbit is not considered good enough to be used by the other processes in the computation chain, and cyan when measurements are present and used by the filter. 


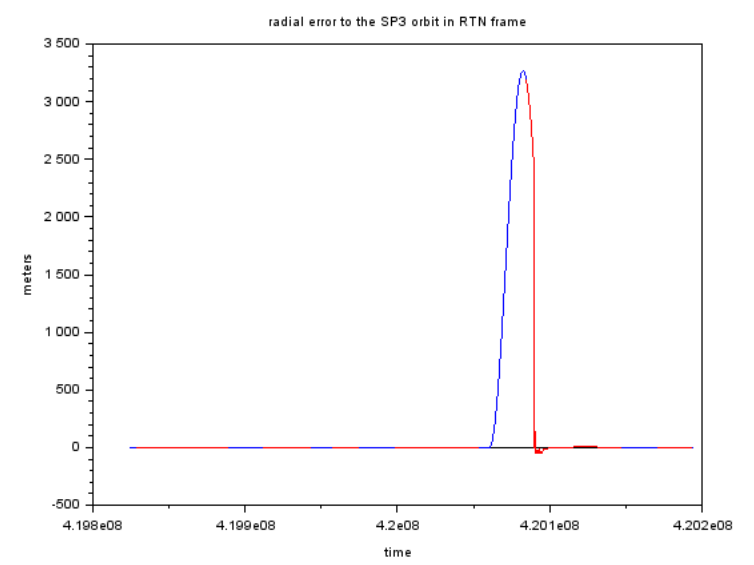

Figure 2: Radial error with respect to the SP3 for a maneuvering satellite

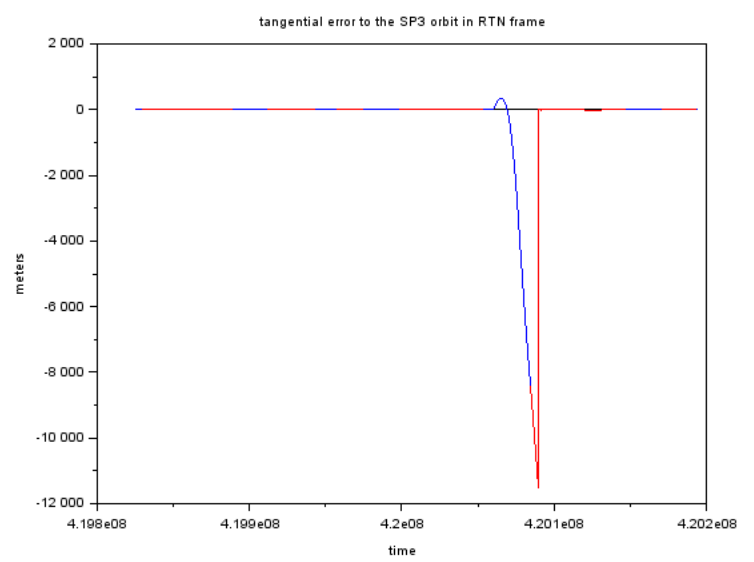

Figure 3: Tangential error with respect to the SP3 for a maneuvering satellite

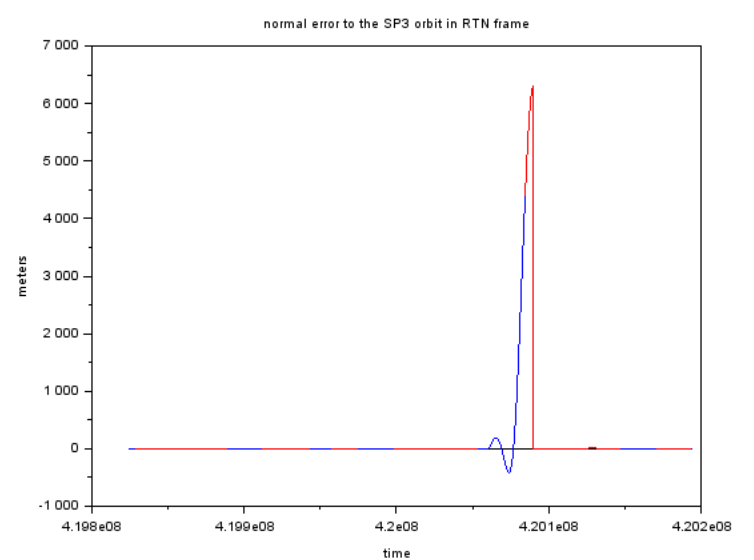

Figure 4: Normal error with respect to the SP3 for a maneuvering satellite

The filter reacted when the satellite came back in view, by comparing the measurements with the propagated orbit. As a consequence, the orbit has quickly reconverged to its true value, which can be seen more precisely below. The Figure 6 shows a zoom of Figure 2 after the maneuver detection.

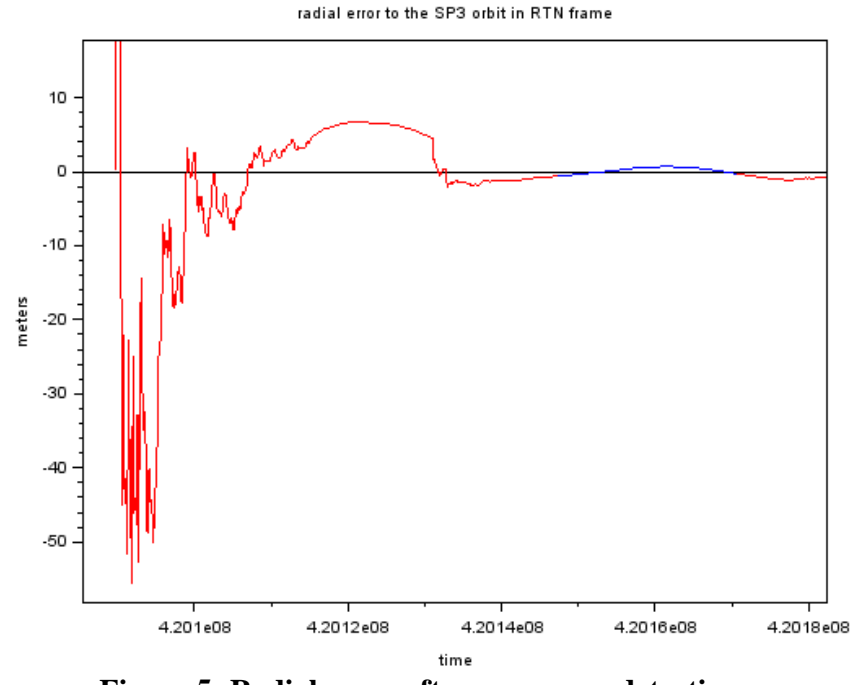

Figure 5: Radial error after maneuver detection

Although the satellite was more than $10 \mathrm{~km}$ away from its propagated position when it comes back in view, the estimated orbit after initialization is within 10 meters in only 3 hours. A precision of one meter was achieved 12 hours after the detection.

\section{NEW TIME SYNCHRONISATION DETERMINATION FOR EGNOS}

This function is in charge of the satellite and stations clock synchronization in order to produce continuous clock corrections from the GPS satellites allowing a precise receiver positioning. In addition, these corrections define implicitly an internal time scale, which must remain close to the GPS time.

\section{A. Measurements and models}

A simple approach would be to solve the clocks independently at each epoch, in order to deal with possible anomalies (jumps). However, the stability needed for the estimation of the satellite clocks implies that the pseudo-range is not sufficient to have correct estimations, due to the measurement noise. So it is necessary to constrain the clock variations from one epoch to the other. This is achieved using the phase measurements.

The process uses the iono-free pseudo-range and phase observables which have been preprocessed in order to remove the possible cycle slips. The tropospheric delays are also corrected. The clock corrections must be consistent with the user models: for example the tropospheric correction must be defined in the same way as for the user (MOPS definition [1]). The measurement equations are for epoch $k$ :

$$
\begin{aligned}
& m=(\rho+T)+h_{i}-h^{j}+\varepsilon \\
& d l=d(\rho+T)+d h_{i}-d h^{j}+\varepsilon
\end{aligned}
$$

The notations are the same as for equation (1). The operator $d$ represents the difference between the value at 
epoch $\mathrm{k}$ and the corresponding value at epoch $\mathrm{k}-1$ (same satellite, same station). $d l$ is the phase variation measured between epoch k-1 and k. It is constructed only if the phase is available at the two epochs, without cycle slip. For the distance computations, the orbits are frozen. The only adjusted parameters are the clock offsets.

\section{B. Filter}

As in the case of the orbit determination filter, the equations (2) can be solved globally in a least squares filter, for a given time span. The structure of the equations allows an efficient recursive least squares formulation: looking at equations (2) we see that now the measurements at epoch $\mathrm{k}$ are dependent from the clocks values at epoch $\mathrm{k}$ and $\mathrm{k}-1$. So the state vector at epoch $\mathrm{k}$ must contain the vectors of the clocks offsets $h_{i}$ and $h^{j}$ at epochs $\mathrm{k}$ and $\mathrm{k}-1$. When all measurements of epoch $\mathrm{k}$ are processed (equations (2)), the part of the state vector corresponding to epoch $\mathrm{k}-1$ is no longer needed for future measurements and is removed. A new set of values is initialized for epoch $\mathrm{k}+1$.

Then, to allow a continuous operation as for the orbit determination, an exponential weighting is applied on the past measurements information matrix. The weighting coefficient is optimized in order to have a smooth solution (equivalent to the definition of the time span of the least squares data set).

As explained for the orbit determination equations, the system of equations (2) has also un-observable corresponding to each continuous clock section (values connected with the phase measurements). As for the orbit determination case, loose constraints to the GPS messages values are added as measurement equations at each epoch, directly on the values of $h^{j}$ participating to a measurement. If there is no measurement on a station or on a satellite, the corresponding clock is undefined (no clock model is used).

Finally the clock solution, which is aligned on the ionofree pseudo-range combination, is aligned on the L1 frequency band (no bias to apply for L1 processing). These satellites clock offsets are used to prepare the fast clock corrections for the SBAS legacy services.

\section{Performances}

The performances results that are provided in this section have been reached using IGS ephemeris to remove the geometry part from the measurements and preparing the system of equations. The rationale is to assess the performances independently from the one provided by the Thales orbit determination module.

The figure [Figure 6] shows the Allan variance of the filter solution with respect to the IGS reference solution. For this computation, only satellite clock values with formal covariance below one meter have been considered.

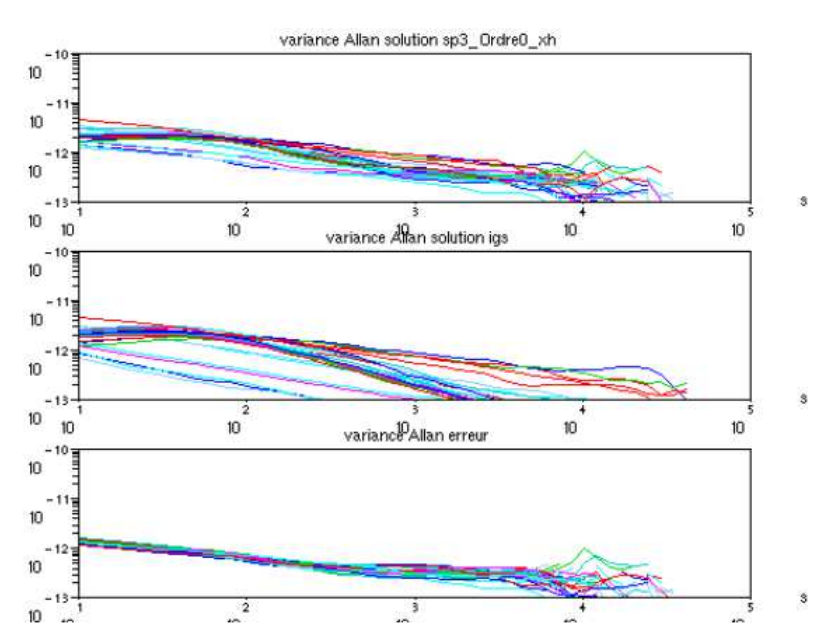

Figure 6: Satellite clock Allan Variance, IGS solution, differences

This results show that the filter is efficient up to $1000 \mathrm{~s}$. From these curves the clocks solution error Allan's deviation is around $10^{-12}$ at $120 \mathrm{~s}$ leading to $7 \mathrm{~cm}$ of possible deviation for a prediction up to $120 \mathrm{~s}$. This performance is fully compatible with the needs of the SBAS mission.

The following figures show the stations clock computed by the filter compared with the result using the code measurements only (green color). The code clock solution have been globally aligned on the one provided by the filter by steering the average of the discrepancies to 0 to each date. The covariance of the solution is presented below.

The figure [Figure 7] presents the behavior of the clock of the station Alberg that is a Rubidium. The measurements provided by the station are quite good qualities although we observe at a time a complete loss of the phase measurement by the receiver. This event involves an automatic complete re-initialization of the clock computation. The convergence is very fast.
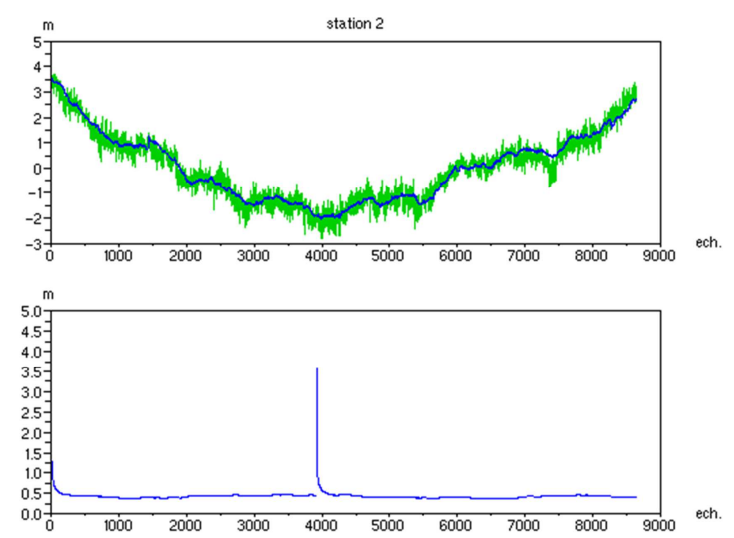

Figure 7: Clock bias for station Alberg (Rb)

Other station results have a significantly worse behavior as it is the case for Sofia station [Figure 8] that is a Cesium. This station provides several code-phase 
inconsistencies involving several re-initializations of the associated clock parameter as it is underlined by the covariance values. However the filter process allows a correct reconstruction of the clock behavior.
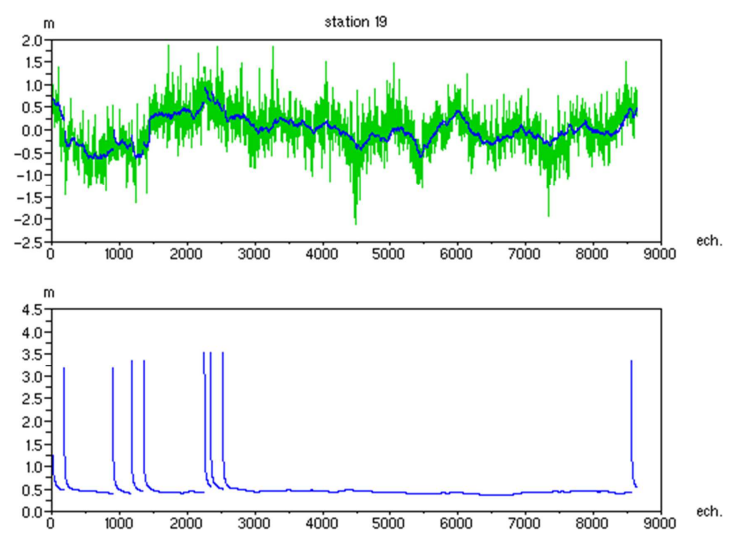

Figure 8: Clock bias for station Sofia (Cs)

Regarding the clock satellite the comparison with the clock computed using code measurement only underlines the interest to use the phase measurements. Indeed at the beginning and the end of visibility the code measurement quality is strongly degraded. However the solution given by the filter provides a good estimate of the clock. The following case [Figure 9] presents an interruption at the middle of the pass. The covariance is reinitialized and the convergence is very fast after the interruption because the satellite is seen by a lot of stations. Nevertheless the visibility angles were roughly low.

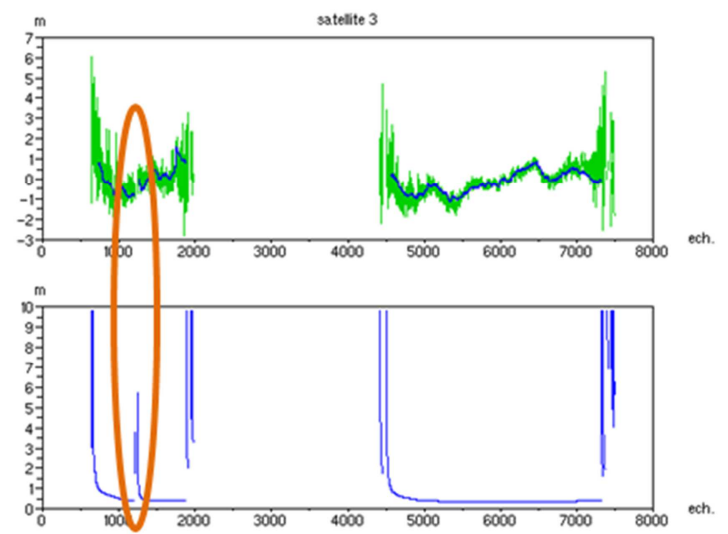

Figure 9: Clock solution for PRN03 and code solution only

The following figure [Figure 10] show the differences between the L1-clock solution given by the filter (steered on the L1 frequency as it broadcasted by the SBAS) and the iono-free IGS solution. Clearly there are biases (due to the reference used at IGS, which is the iono-free combination of $\mathrm{C} 1 \mathrm{P}, \mathrm{C} 2 \mathrm{P}$, instead of $\mathrm{C} 1 \mathrm{C}$ in our solution) but the solution computed by the filter does not produce any jump in case of arrival or the leave of a satellite in the clock problem.
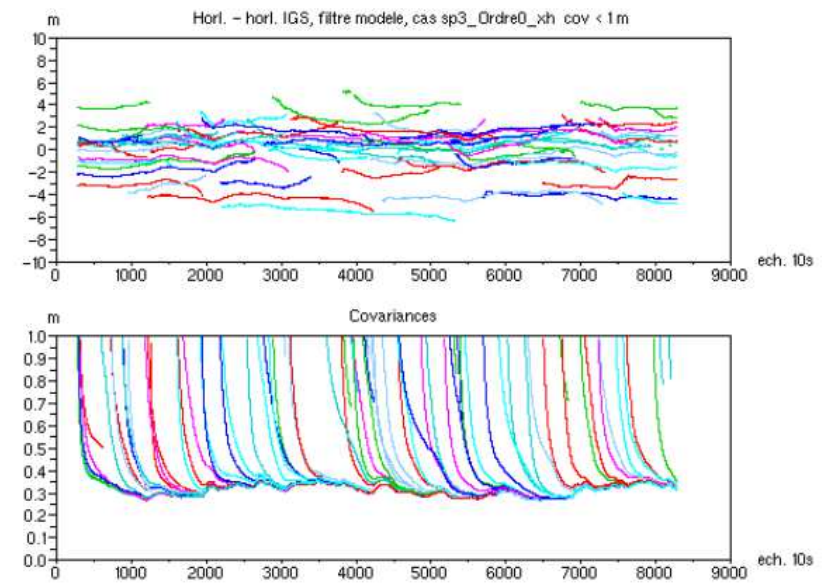

Figure 10: IGS clocks comparison, all satellites, gap values and formal covariance

The time synchronization filter is not perturbed by the raising and setting of satellites above the visibility area. It is thus properly adapted for SBAS context.

\section{RESULTS AND DISCUSSION}

Hereafter are presented the results of the comparison of orbit and clock corrections provided by different SBAS (EGNOS, WAAS, GAGAN and MSAS) with respect to IGS results, as well as the performance of the new algorithms.

Figure 11 shows the comparison between different SBAS for the orbit error on PRN 6, on September $4^{\text {th }} 2015$. The same kind of results is obtained with the other PRNs.

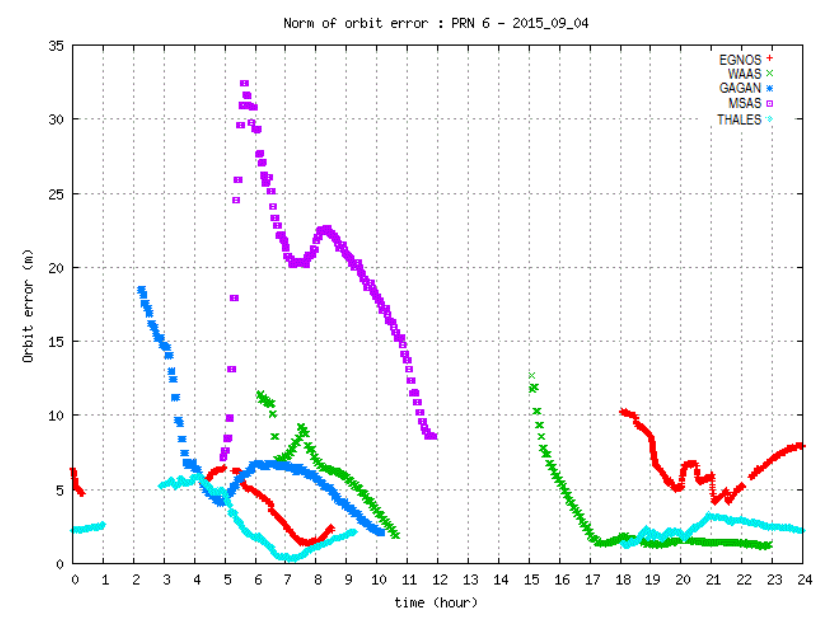

Figure 11: Comparison of the 3D orbit error for PRN 6, on September $4^{\text {th }} 2015$

The plots are discontinuous since the orbits were reconstructed with the slow correction messages broadcasted by the different SBAS. The messages are available only when the satellite is in view of the SBAS station network. This is also why the curves are not on the same time span.

The Thales orbit determination process gives a much better orbit than the ones from the other SBAS, in 
particular better than the EGNOS one. It is more straightforward to compare these two solutions, as the Thales solution was built using the same EGNOS RIMS station network.

Figure 12 is the clock error with respect to the IGS results for all SBAS.

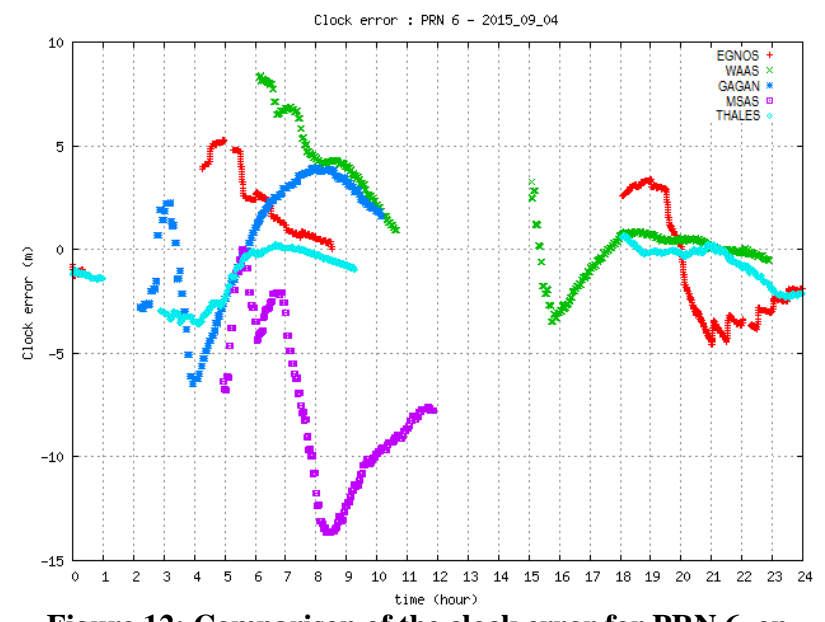

Figure 12: Comparison of the clock error for PRN 6, on September $4^{\text {th }} 2015$

Again, the clock output by the Thales solution is similar or better than the ones from the other SBAS. The precision is metric and the solution is more stable. This result highlights the good level of orbit and clock decorrelation provided by the different filters and is an indicator of their internal performances. We recall however that, regarding the user navigation performance, the most important is the quality of both orbit and clock corrections taken together and not the independent quality of orbit correction and clock correction taken separately.

Figure 13 shows the effects of the orbit and clock calculated from a user point of view. An SBAS must protect the users in its service area by broadcasting a UDRE value, that must bound the projection of the orbit and clock error for a satellite along the line of sight. The user in the service area with the biggest error is called the WUL (Worst User Location) and its error is the SREW (Satellite Residual for the Worst user location). The figure then shows the value of the SREW with the SBAS solutions.

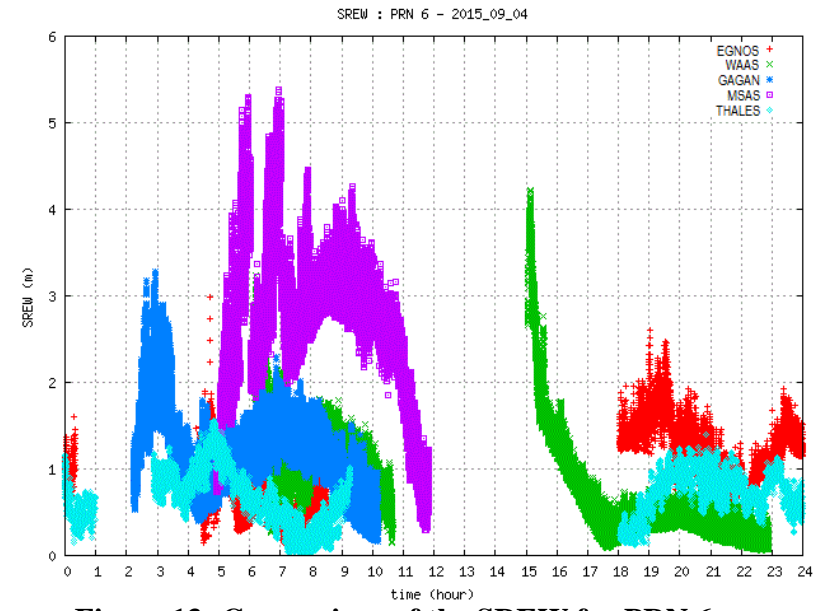

Figure 13: Comparison of the SREW for PRN 6, on September $4^{\text {th }} 2015$

The better orbit and clock for the Thales solution translates into lower values of the SREW. The Root Mean Square of the SREW is around $0.70 \mathrm{~m}$. Having a lower SREW allows an SBAS to transmit lower values of UDRE while keeping the same level of integrity. This, in turn, will expand the different service levels availability areas.

\section{CONCLUSIONS AND PERSPECTIVES}

A new module for the orbit and clock determination of GNSS satellites has been presented. It is designed implemented in a real time formulation, ideal in an SBAS context.

The orbit and clock determination module shows significant improvements in terms of precision compared with the other SBAS, as well as a strong ability of detecting and reacting to a satellite manoeuvre. The convergence time after a satellite is detected as performing a manoeuvre is only a few hours of visibility.

The improved orbits and clocks calculated result in a lower SREW in the service area, thereby allowing to increase the integrity and availability levels. Lower UDRE values can be broadcasted by the SBAS for the same integrity level, an effect that will be noticeable particularly for dual frequency users.

\section{REFERENCES}

[1] Minimum operational performance standards for Global positioning system / wide area augmentation system airborne equipment, DO-229D rev 1, Jan. 2013, RTCA ed. Washington, DC.

[2] Stochastic Processes and Filtering Theory, A. H. Jazwinski

[3] A guide to using international GNSS service (IGS) products. Jan Kouba.

[4] Statistical Orbit Determination, Byron D. Tapley 